\title{
Does tranexamic acid improve outcomes in traumatic brain injury?
}

In this Practice article (BMJ 2016;354:i4814, doi:10.1136/bmj. i4814), Abda Mahmood and colleagues, an error occurred throughout Figure 1. The label "II-PA" should read "TPA.” We apologise for any confusion this may have caused. 